\title{
Biochemical and Molecular Resistance Mechanisms to Dichlorodiphenyltrichloroethane, (DDT) and Some Pyrethroid Insecticides in Vector of West Nile Virus, Culex Pipiens
}

\author{
Yaser Salim Abadi ${ }^{1,2}$, Hassan Vatandoost*1,3, Mohammad Ali Oshaghi ${ }^{1}$, Mohamad Reza Abai ${ }^{1}$, \\ Ahmad Ali Enayati ${ }^{4}$ and Mohammad Amin Gorouhi ${ }^{5}$ \\ ${ }^{1}$ Department of Medical Entomology and Vector Control, School of Public Health, Tehran University of Medical Sciences, Tehran, \\ Iran
}

${ }^{2}$ Department of Health Services and Health Promotion, School of Health, Rafsanjan University of Medical Sciences, Rafsanjan, Iran ${ }^{3}$ Department of Chemical Pollutants and Pesticides, Institute for Environmental Research, Tehran University of Medical Sciences, Tehran, Iran

${ }^{4}$ School of Public Health and Health Sciences Research Centre, Mazandaran University of Medical Sciences, Sari, Iran

${ }^{5}$ Department of Vector Biology and Control, School of Health, Kerman University of Medical Sciences, Kerman, Iran. Department of Medical Entomology and Vector Control, School of Public Health, Tehran University of Medical Sciences, Tehran, Iran

*Corresponding author: Hassan Vatandoost, Department of Medical Entomology and Vector Control, School of Public Health, and Department of Chemical Pollutants and Pesticides, Institute for Environmental Research, Tehran University of Medical Sciences, Tehran, Iran

\section{ARTICLE INFO}

Received: May 11, 2021

Published: 杫 June 02, 2021

Citation: Yaser Salim A, Hassan V, Mohammad Ali O, Mohamad Reza A, Ahmad Ali E, et al., Biochemical and Molecular Resistance Mechanisms to Dichlorodiphenyltrichloroethane, (DDT) and Some Pyrethroid Insecticides in Vector of West Nile Virus, Culex Pipiens. Biomed J Sci \& Tech Res 36(2)-2021. BJSTR. MS.ID.005817.

Keywords: Cx.pipiens; Insecticides Resistance; Molecular; Biochemical Mechanisms

Abbreviations: GST: Glutathione-S-Transferases; MFO: Mixed Function Oxidase; RR: Resistance Ratio; PCR: Polymerase Chain Reaction; Dichlorodiphenyltrichloroethane

\section{ABSTRACT}

Culex pipiens complex act as an important vector of several vector-borne diseases such as filariasis, West Nile virus, Japanese encephalitis and bird malaria. This study was designed in order to clarify the molecular and biochemical resistance mechanisms in Cx.pipiens to Dichlorodiphenyltrichloroethane, (DDT) and some pyrethroid insecticides from Tehran, capital of Iran. Profile activities of $\alpha$ - and $ß$-esterases, Mixed Function Oxidase (MFO), Glutathione-S Transferase (GST), were tested for Cx.pipiens strain with resistance ratio of 85.75 to Lambdacyhalothrin and also about DDT resistant strain in comparison with Lab strain. In the present research a molecular study also performed on both lambdacyhalothrin and DDT. Resistant strains for detection of the mutation in the sodium channel gene which is associated with (knockdown) kdr insecticide resistance to pyrethroid and DDT. For comparison of average between two groups T test were used.

Our finding showed that there are significant different $(\mathrm{p}<0.05)$ between the mean activity of $\alpha$-, $\beta$-esterases and (MFO), in both lambacyhalothrin and DDT resistant strain in comparison with Laboratory Strain (Lab-strain), but there is no significant difference $(p>0.05)$ about (GST) in the both strain in comparison with Lab-Strain. Molecular study for detection of L1014F or L1014S mutation in sodium channel gene showed lack of the mutation responsible for insecticide resistance to pyrethroid and DDT. This study showed that the resistance to pyrethroids and DDT in the Cx.pipiens is enzymatic, but not targets site insensitivity of sodium channel gene. Findings of this research could provide a clue for logical operations of future chemical control program. 


\section{Introduction}

Culex pipiens complex is a worldwide species and among its members, Cx.pipiens pipiens and $C x$. quinquefasciatus are two more important vectors of several vector-borne diseases regarding to different group of pathogens which causing filariasis, West Nile virus, Japanese encephalitis and bird malaria [1-4]. Culex genus habitat mainly is sewage system of cities and due to this lifestyle resistance to the most group of insecticides [5-8]. Based on finding of many studies around the world as evaluating resistance status of Cx.pipiens to insecticides, result indicated that this species has multiple insecticide resistances or at least resistance to one group of insecticides, moreover during recent years resistance to pyrethroids insecticides and DDT is spreading around the world [914]. According to previous studies in Tehran related to evaluation of susceptibility and Irritability level to insecticides which performed on Cx.pipiens complex, indicated that resistance ratio in $C x$. pipiens to different groups of insecticides increased and also it can be concluded, this species is quite resistant to DDT $[15,16]$.

In the most species of mosquitoes, resistance to different groups of insecticides caused by two main mechanisms which known as metabolic resistance due to enzymatic detoxification by enzymes involved in resistance including cytochrome P450 oxidases, esterases and Glutathione-S-Transferases (GST) and also it seems that resistance to pyrethroid compounds caused by increasing level of oxidases and second reason is target site insensitivity due to substitution in nucleotides about pyrethroid compounds and DDT. Resistance cases this mutation lead to translating leucine to phenylalanine or leucine to Serine in the S6 hydrophobic segment of domain II in the sodium channel gene that known as Knock down resistance (kdr) mutation $[17,18]$. In some species from Culex genus the same kdr mutation is present at position 1014 which known as L1014F to (TTA to TTT) orL1014S (TTA to TCA) in pyrethroidresistant cases $[13,18]$. The present study was performed for clarifying the molecular and biochemical resistance mechanisms in Cx. pipiens to DDT and some pyrethroid insecticides.

\section{Materials and Methods}

\section{Study Area}

This study was performed in Tehran city $\left(35^{\circ} 41^{\prime} 46^{\prime \prime} \mathrm{N}, 51^{\circ} 25^{\prime}\right.$ $23^{\prime \prime}$ E), Tehran Province, the capital of Iran (Figure 1).

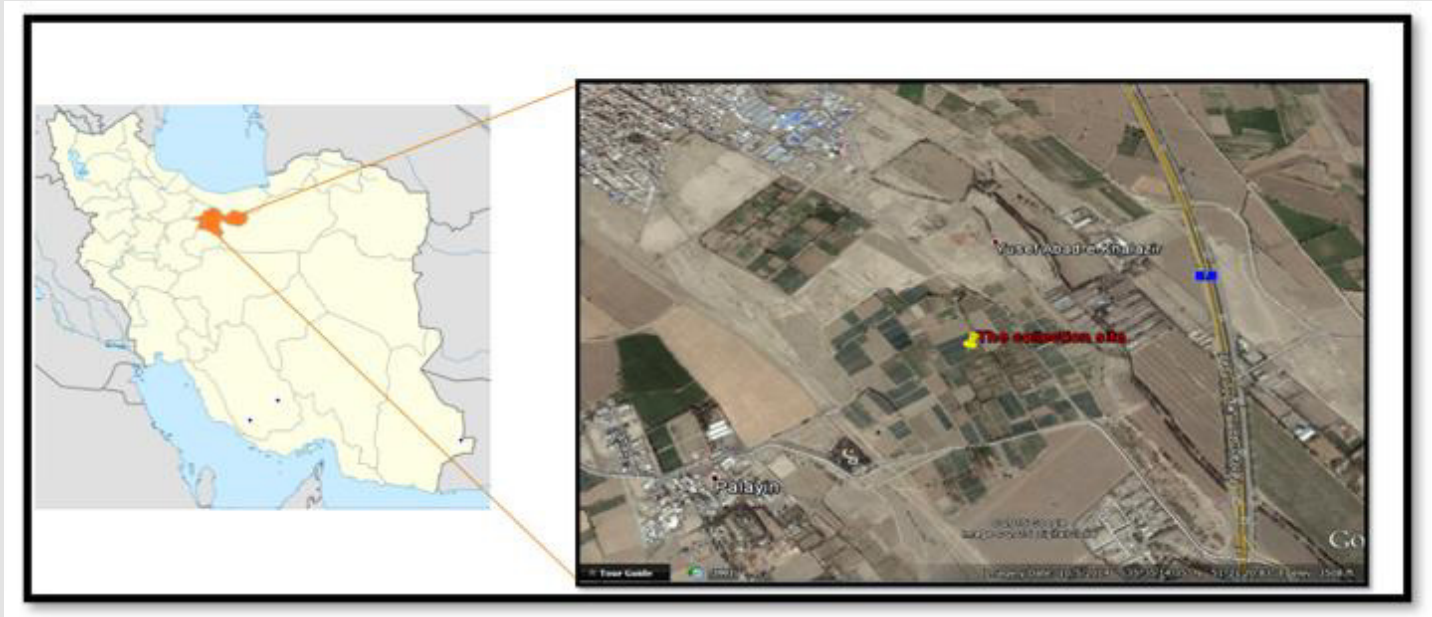

Figure 1: Map of study area in Tehran city and the collection site, Iran.

\section{Mosquito Strains}

The wild larval stage strains of $C x$. pipiens collected from collection site in the study area and for more investigation all wild -caught larval were reared in the insectary of School of Public Health, Tehran University of Medical Sciences under the standard condition. Moreover, as comparison the laboratory strain of $C x$. pipiens were used.

\section{Insecticides}

In the following experiment for biochemical and molecular assays in $C X$. pipiens four Insecticides including: DDT $4 \%$, Lambdacyhalothrin $0.05 \%$, Deltamethrin $0.05 \%$, Cyfluthrin 0.15 $\%$ were used according to World Health Organization (WHO) standard method [19]

\section{The Process for Selection of Insecticides-Resistant Population}

A criterion for selection of insecticides-resistant population is the highest Resistance Ratio (RR) about pyrethroids insecticides tested. Selection process carried out on adult by exposing with a time that leads to about $50-70 \%$ mortality. In this study due to highest resistance ratio about Lambdacyhalothrin, for attaining a homogenous resistant population, 3 generation after the initial population with more than 80 -fold resistant were used. Moreover because of wild strain with 1 hours exposure to DDT and during a period of 24 hours after recovery yielded no mortality, both of these population selected for biochemical and molecular studies. 


\section{Biochemical Assays}

Forty mosquitoes from three population including: pyrethroid insecticides that have highest resistance ratio (RR) which exposed to insecticide (resistant to Lambdacyhalothrin), resistant to DDT and also lab strains were evaluated for Mixed Function Oxidase (MFO), Glutathione-S-Transferase (GST), $\alpha$-and ß-esterases and protein assay. Measurement of enzymes activity generally carried out in accordance with the standard method [20]. At the first, adult female of $C x$. pipiens was individually homogenized along with 200 microliters of distilled water in flat-bottomed micro plates which putted on ice. For all biochemical assays, each individually homogenized mosquito was transferred to two wells on a 96 well flat-bottomed micro plate, by a microplate reader (ELX808 Ultra Microplate Reader BIO-TEK ${ }^{\circledR}$ ) after adding buffers and reagents to homogenized according to protocol. Absorbance levels with the use of wavelength specific for each enzyme were measured [20]. Moreover, as the correction of errors related to volume of mosquito homogenates due to different protein content from each mosquito. Absorbance values for mosquito were corrected finally the means of enzyme activities for each mosquito's strain were compared with the Lab strain by Unpaired t-test, Mann-Whitney test and P-Value $<0.05$ was considered as significance level.

\section{Molecular Study of Resistance}

In the present research a molecular study also performed on both lambdacyhalothrin and DDT resistant strains for detection of the mutation in the sodium channel gene which is associated with kdr insecticide resistance to pyrethroid insecticides and DDTG genomic DNA related to each three strains was extracted by Collins method [21]. For amplifying a 521 base pair (bp) fragment of the sodium channel gene by polymerase chain reaction(PCR) which containing the region that kdr mutations occur, primers: $\mathrm{Cpp} 1(5 /$ CCT GCC ACG GTG GAA CTT C3/) andCpp2 (5/GGA CAA AAG CAA GGC TAA GAA3/) according to thermal cycling conditions: 30 cycles of $94^{\circ} \mathrm{C}$ for $40 \mathrm{~s}, 60^{\circ} \mathrm{C}$ for $50 \mathrm{~s}$ and elongation at $72^{\circ} \mathrm{C}$ for $40 \mathrm{~s}$, followed by final extension at $72^{\circ} \mathrm{C}$ for 8 minute were used. Bioinformatics soft wares such as Clustal W2 and Blast were used for sequence alignment also for Translation nucleic acids to amino acids ExPASy

was used.

\section{Results}

\section{Selection Process}

In the present study after detection the highest resistance ratio (RR) about pyrethroids insecticides tested, selection process performed on adult and after 3rd selection a Lambdacyhalothrin resistant population of Cx.pipiens was achieved with 85.75 -fold resistance ratio at Lethal Time 50\% (LT50) level.

\section{Biochemical Assays}

Profile activities of $\alpha$ - and $\beta$-esterases, Mixed Function Oxidase (MFO), Glutathione-S-Transferase (GST), were tested for two Cx.pipiens populations with resistance ratio of 85.75 to Lambdacyhalothrin and resistant to DDT population in comparison with Lab strain. The results are summarized in Table 1 which shows the median level of enzymatic activity related to all three populations.

\section{Mixed Function Oxidase (MFO)}

In both DDT and Lambdacyhalothrin resistant populations in comparison to Lab strain, results showed that there is significant difference in MFO activity levels $(\mathrm{P}<0.05)$ (Table 1) (Figure 2).

\section{Statistical Analysis}

For comparison of average between two groups $\mathrm{T}$ test were used

\section{a) Glutathione-S-transferase (GST)}

Statistical analysis indicated that, GST activity in the two populations was not significantly different from that of the Labstrain (P value $>0.05$ ) (Table 1) (Figure 2).

\section{b) $\alpha$ and B-Esterase}

In the present study both DDT and Lambdacyhalothrin resistant populations, the median activity levels of $\alpha$ and $\beta$-EST were significantly different from the Lab strain $(\mathrm{P}<0.05)$ (Table 1) (Figure 2).

Table 1: Quantification of enzymatic activity of MFO, GST, a and $\beta$-esterase in three populations (resistant to Lambdacyhalothrin, resistant to DD.T and lab-strian) of Culex pipiens.

\begin{tabular}{|c|c|c|c|c|c|c|c|c|}
\hline \multirow{2}{*}{ Strains } & \multicolumn{2}{|c|}{ MFO (EU Cyt.p450/mg) } & \multicolumn{2}{|c|}{ GST (mM/min/mg) } & \multicolumn{2}{c|}{$\boldsymbol{\alpha}$-esterase $(\boldsymbol{\mu M} / \mathbf{m i n} / \mathbf{m g})$} & \multicolumn{2}{c|}{$\boldsymbol{\beta}$-esterase $(\boldsymbol{\mu M} / \mathbf{m i n} / \mathbf{m g})$} \\
\cline { 2 - 9 } & Na & Median b & Na & Median b & Na & Median b & N a & Median b \\
\hline Lab & 40 & -0.000019 & 40 & 0.06857 & 40 & 0.0001 & 40 & 0.00034 \\
\hline Resistant to DDT & 40 & 0.000021 & 40 & 0.11485 & 40 & 0.00061 & 40 & 0.00062 \\
\hline $\begin{array}{c}\text { Resistant to } \\
\text { Lambdacyhalothrin }\end{array}$ & 40 & 0.000036 & 40 & 0.13222 & 40 & 0.00073 & 40 & 0.00048 \\
\hline
\end{tabular}

Note: a-Number of mosquitoes tested.

b-Median value for each enzymatic activity

Copyright@ Hassan Vatandoost | Biomed J Sci \& Tech Res | BJSTR. MS.ID.005817. 


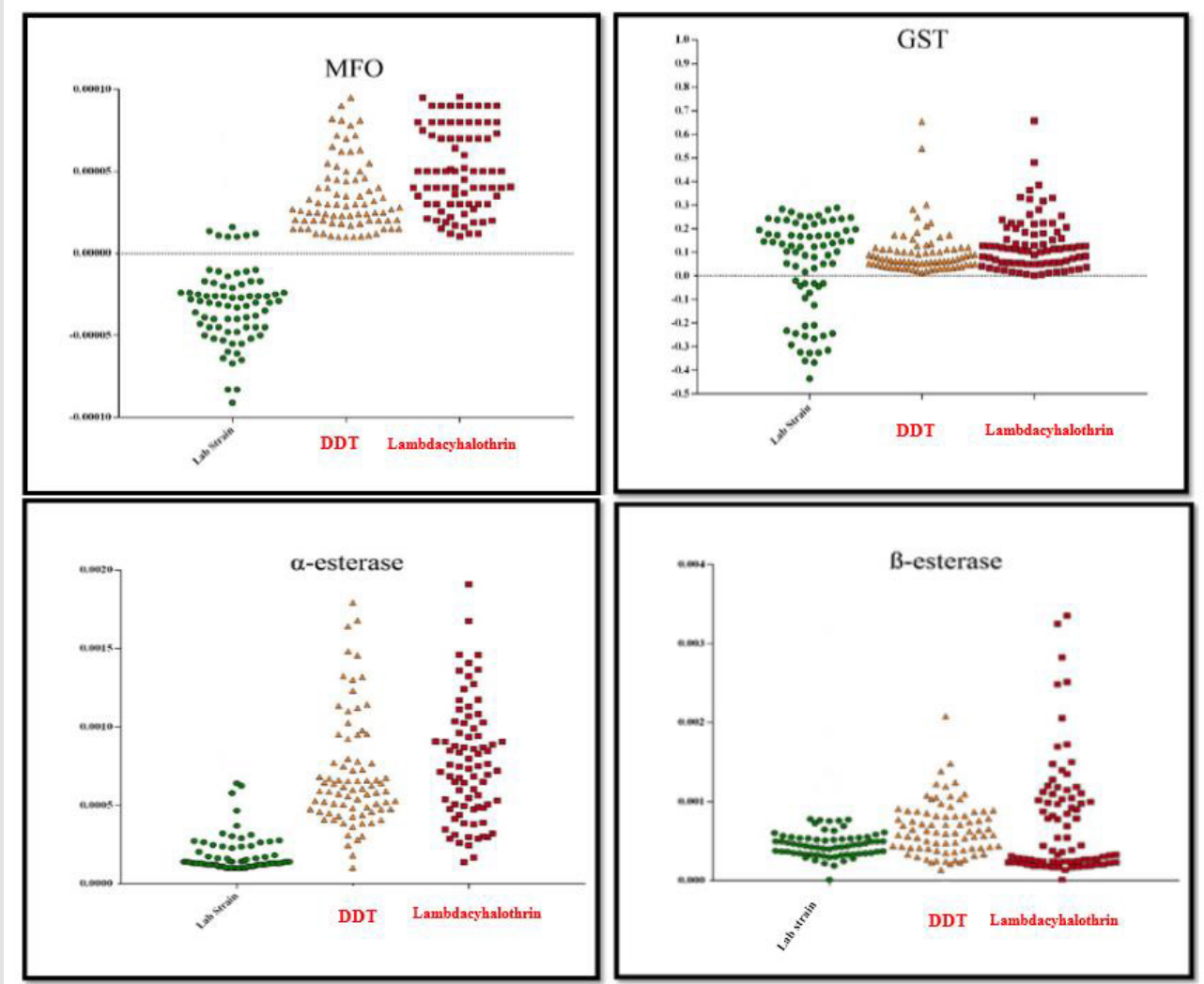

Figure 2: Activity profile of MFO, GST and a and B-Esterase enzymes in two populations (resistant to Lambdacyhalothrin and resistant to DDT compared with lab-strain of Culex pipiens.

\section{Amplification and Sequencing of Sodium Channel Gene Fragments in Cx. Pipiens}

A 521-bp fragment of the sodium channel gene from all three populations by Polymerase Chain Reaction (PCR) using primers: Cpp1 (5' CCT GCC ACG GTG GAA CTT C3') andCpp2 (5'GGA CAA AAG CAA GGC TAA GAA3') were amplified (Figure 3). From each Cx.pipiens population 40 samples of sodium channel gene fragment was sequenced. By using Bioinformatics software such as Clustal W2 and Blast all sequencing results were checked in order to ensure that results are valid. Moreover, as comparing sequencing results of this study with other sequences recorded in Gen Bank by Blast result showed that our sequence is more similar to Culex pipiens pallens (Accession number: GU198941.1). More investigation of kdr region showed that: L1014F substitution due to changing TTA codon to TTT and L1014S substitution (TTA to TCA) causing resistance in the kdr gene was not observed. Moreover, in all three population in the mentioned region, similar Culex pipiens pallens (Accession number: GU198941.1), TTA codon was exist (highlighted in yellow) (Figure 4).

According to ExPASy results related to translating nucleic acids to amino acids, sequences of Cx.pipiens sodium channel gene were compared with other similar amino acid sequences of mosquitoes were available in the gene bank. By comparing result of current study with the most similar sequences related to Cx.pipiens pallens results revealed that in the region with possibility $\mathrm{Kdr}$ mutation at position 1014 in the sodium channel gene due to translation leucine to phenylalanine or serine There is no mutation and this fact, confirmed lack of Kdr mutation in two Cx.pipiens populations. Except a substitution due to changing TTA codon to TTT in the exonI (highlighted in gray) resulted in single amino acid substitution of leucine to phenylalanine in Culex pipiens pallens, all other amino acids were quite similar and also These differences, may be considerable to insecticide resistance in the future about this species (Figure 4). 


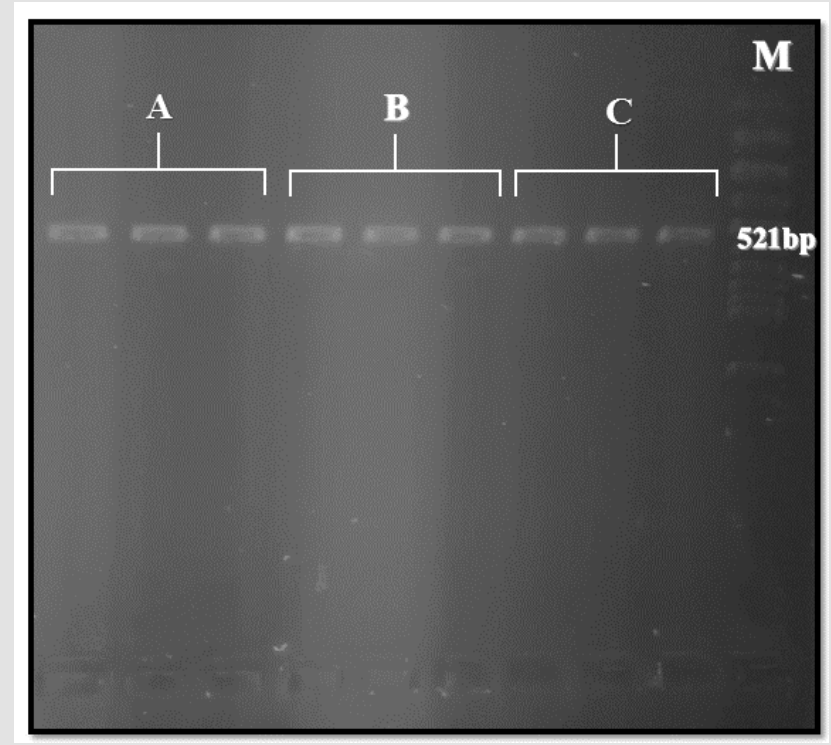

Figure 3: Amplification of sodium channel gene (521bp) in Cx.pipiens from three different populations -A:Lab-Strain,B:resistant to Lambdacyhalothrin,C:resistant to DDT.M: 100 bp ladder.

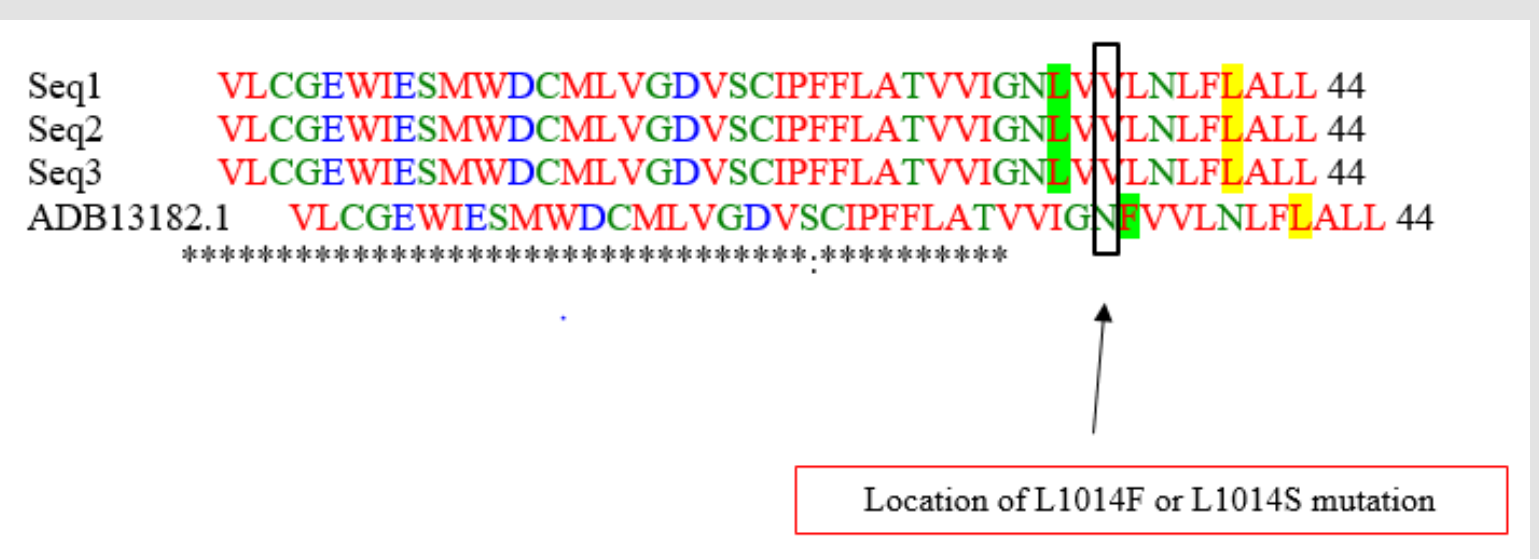

Figure 4: Comparison of translated sodium channel gene region amino acids sequence of this study (Seq1, Seq2, Seq3) with other similar registered genes in gene bank Culex pipiens pallens (S).

\section{Discussion}

In the present investigation the biochemical and molecular basis of resistance in two population, resistant to DDT and pyrethroids insecticides and Lab strain was conducted. Results showed that there are significant different $(\mathrm{p}<0.05)$ between the mean activity of $\alpha$ and $\beta$-esterase and (MFO), in both lambacyhalothrin and DDT resistant population compared with Lab-Strain. However, there is no significant difference $(p>0.05)$ about (GST) in the both populations compared with Lab-Strain. Molecular study for detection of L1014F or L1014s mutation in sodium channel gene showed lack of the mutation responsible for insecticide resistance which lead to cross-resistance between pyrethroids insecticides and DDT in Cx pipiens populations. Based on our finding, it seems that metabolic mechanisms due to increased enzyme levels have considerable role in resistance to pyrethroid insecticides and DDT in $C x$ pipiens populations and the main reason for increased resistance to insecticides are MFO, $\alpha$ and $ß$ esterase.

Although there was no significant different $(p>0.05)$ about (GST) in the both populations compared with Lab-strain. We cannot definitively ignore the important role of GST in resistance to organochlorines and pyrethroids by detoxification of them [2223]. One of the reasons which can explain that why this difference was not significant is moderate resistance to DDT in lab strain of Cx.pipiens. There are many reports about metabolic resistance to DDT and pyrethroid insecticides due to increase level of enzymes in medically important vectors of mosquitos, especially in Culex genus. MFO as one of the most influential enzymes alone or with other enzymes responsible for biochemical resistance mentioned as a majority reason for resistance to Pyrethroid insecticides and 
DDT [24-27]. Moreover, in some species more than one enzyme introduced as an enzyme responsible for resistance to DDT and Pyrethroid insecticides.

In resistant to DDT and Pyrethroid insecticides populations of Aedes aegypti from Trinidad and Tobago after biochemical surveying for detection of resistance mechanism result showed that elevated levels of $\alpha$-esterase and MFO enzymes in all Ae. aegypti populations were present also approximately in all populations increased levels of leand GST levels were indicated 27 Activities of esterases, monooxygenases in Anopheles stephensi from Dubai (DUB-R) resistant to permethrin were measured and result showed that level of all enzymes in resistant strain comparing with susceptible strain were significantly higher [28]. In Cx.quinquefasciatus mosquitoes from Benin related to four different localities ,resistant to DDT and permethrin in three areas were detected and also higher levels of $\alpha$ and $\beta$ and GST activity in field collected compared with susceptible strain [12]. In our study except GST activity which there was no significant difference between two population in compression of Lab strain, result about other enzymes activity level was in parallel to three mentioned studies about resistant to DDT and Pyrethroid insecticides in mosquitoes $[12,28]$.

Molecular study for detection probable mutations at position 1014 in sodium channel gene showed lack of the mutation responsible and also by translation nucleic to amino acids result indicated that mutation in two Cx.pipiens population due to translation leucine to phenylalanine or serine was not exist and with emphasis to this finding we can describe there are no Kdr mutation in our field collected populations of Cx.pipiens. Based on some studies about resistance to DDT and Pyrethroid insecticides. High insecticide resistance correlated with mutations at position 1014 in sodium channel gene Which is known as the kdr mutation indicated in mosquitoes [29,30]. In six out of seven Ppyrethroid insecticides populations of Anopheles gambiae, mutations in sodium channel gene reported as responsible for survival to pyrethroid exposure (Martinez-Torres et al., 1998).

The results of Chen et al. study (2010) showed that high L1014F mutation frequency in six populations of $C x$. pipens pallens caused high prevalence of pyrethroid resistance in Eastern China [13]. In another study two alternative kdr alleles, both resulting in a L1014F substitution were detected in some pyrethroid insecticides and DDT resistant strains of $C X$. quinquefasciatus [14]. Multiple insecticide resistance mechanisms in An. gambiae and Cx. quinquefasciatus in have been reported, also in permethrin and DDT resistant populations of An. gambiae and $C x$. quinquefasciatus this resistance to insecticides attributed to presence of target site insensitivity due to kdr mutation [12]. Finally according to our finding there are any evidence for molecular bases of resistance to pyrethroid insecticides and DDT due to kdr mutation in Cx.pipiens. It seems that biochemical mechanism through enzymes activity may be lead to this highly level resistance to insecticides in Cx.pipiens.

\section{Conflict of Interest}

The authors declare that there is no conflict of interest.

\section{Acknowledgement}

This study was financially supported by the Deputy for Research, Tehran University of Medical Sciences, and Code No: 93-01-2723053, as well as the Ministry of Health and Medical Education, Iran. National Institute for Medical Research Development (NIMAD) under code number of 995633.

\section{References}

1. Smith JL, Fonseca DM (2004) Rapid assays for identification of members of the Culex (Culex) pipiens complex, their hybrids, and other sibling species (Diptera: Culicidae). Am J Trop Med Hyg 70(4): 339-345.

2. Richards SL, Lord CC, Pesko KN, Tabachnick WJ (2010) Environmental and biological factors influencing Culex pipiens quinquefasciatus (Diptera: Culicidae) vector competence for West Nile Virus. Am J Trop Med Hyg 83(1): 126-134.

3. Strickman D, Fonseca DM (2012) Autogeny in Culex pipiens complex mosquitoes from the San Francisco bay area. Am J Trop Med Hyg 87(4): 719-726.

4. Pocquet N, Milesi P, Makoundou P, Unal S, Zumbo B, et al. (2013) Multiple Insecticide Resistances in the Disease Vector Culex quinquefasciatus from Western Indian Ocean. PLoS One 8(10): e77855.

5. Lines JD (1998) Do agricultural insecticides select for insecticide resistance in mosquitoes: A look at the evidence. Parasitol Today 8(4): $17-20$.

6. Vatandoost H (2004) Structure-activity relationship of pyrethroids against different geographical strains of larvae of malaria vector Anopheles stephensi and role of mixed function oxidase in resistance phenomenon. Acta Med Iran 42(2): 89-96.

7. Calhoun LM, Avery M, Jones L, Gunarto K, King R, et al. (2007) Combined sewage overflows (CSO) are major urban breeding sites for Culex quinquefasciatus in Atlanta, Georgia. Am J Trop Med Hyg 77(3): 478-484.

8. Salim Abadi Y, Vatandoost H, Rassi Y, Abaei MR, Sanei Dehkordi AR, et al. (2010) Evaluation of biological control agents for mosquitoes control in artificial breeding places. Asian Pac J Trop Med 3(4): 276-277.

9. Amin AM, Hemingway J (1989) Preliminary investigation of the mechanisms of DDT and pyrethroid resistance in Culex quinquefasciatus Say (Diptera: Culicidae) from Saudi Arabia. Bull Entomol Res 79(03): 361-366.

10. Prapanthadara L, Koottathep S, Promtet N, Suwonkerd W, Ketterman AJ, et al. (1999) Correlation of glutathione S-transferase and DDT dehydrochlorinase activities with DDT susceptibility in Anopheles and Culex mosquitos from northern Thailand. Southeast Asian J Trop Med Publ Health 31: 111-118

11. McAbee RD, Kang KD, Stanich MA, Christiansen JA, Wheelock CE, et al. (2004) Pyrethroid tolerance in Culex pipiens pipiens var molestus from Marin County, California. Pest Manag Sci 60(4): 359-368.

12. Corbel V, N Guessan R, Brengues C, Chandre F, Djogbenou L, et al. (2007) Multiple insecticide resistance mechanisms in Anopheles gambiae and Culex quinquefasciatus from Benin, West Africa. Acta Trop 101(3): 207216. 
13. Chen L, Zhong D, Zhang D, Shi L, Zhou G, et al. (2010) Molecular ecology of pyrethroid knockdown resistance in Culex pipiens pallens mosquitoes. PloS one 5(7): e11681.

14. Jones CM, Machin C, Mohammed K, Majambere S, Ali AS, et al. (2012) Insecticide resistance in Culex quinquefasciatus from Zanzibar: implications for vector control programmes. Parasit Vector 5: 78-83.

15. Nazari M, Janbakhsh B (2000) A survey of the susceptibility level of Culex theileri and Cx.pipiens to DDT, Dieldrin,Propoxur and Malathion in the southern area of Tehran. J Uromia Univ Med Sci 11(1): 13-19.

16. Salim Abadi, Y, Oshaghi MA, Enayati AA, Abai MR, Vatandoost H, et al. (2016) High Insecticides Resistance in Culex pipiens (Diptera: Culicidae) from Tehran, Capital of Iran. J Arthropod Borne Dis 10(4): 483-492.

17. Williamson MS, Martinez Torres D, Hick CA, Devonshire AL (1996) Identification of mutations in the housefly para-type sodium channe gene associated with knockdown resistance (kdr) to pyrethroid insecticides. Mol Gen Genet 252 (1-2): 51-60.

18. Martinez Torres D, Chandre F, Williamson MS, Darriet F, Berge JB, et al (1998) Molecular characterization of pyrethroid knockdown resistance $(\mathrm{kdr})$ in the major malaria vector Anopheles gambiae ss. Insect Mol Biol $7(2): 179-184$.

19. (2013) WHO. Test procedures for insecticide resistance monitoring in malaria vector mosquitoes. World Health Organization, Geneva, Switzerland.

20. Penilla PR, Rodriguez AD, Hemingway J, Torres JL, Arredondo Jiménez JI, et al. (1998) Resistance management strategies in malaria vector mosquito control. Baseline data for a large-scale field trial against Anopheles albimanus in Mexico. Med Vet Entomol 12(3): 217-233.

21. Collins FH, Mendez MA, Rasmussen MO, Mehaffey PC, Besansky NJ, et al. (1987) Rribosomal RNA gene probe differentiates member species of the Anopheles gambiae complex. Am J Trop Med Hyg 37(1): 37-41.

\section{ISSN: 2574-1241}

DOI: 10.26717/BJSTR.2021.36.005817

Hassan Vatandoost. Biomed J Sci \& Tech Res

(C) This work is licensed under Creative Commons Attribution 4.0 License

Submission Link: https://biomedres.us/submit-manuscript.php
22. Xu Q, Liu H, Zhang L, Liu N (2005) Resistance in the mosquito, Culex quinquefasciatus, and possible mechanisms for resistance. Pest Manag Sci 61(11): 1096-1102.

23. Che Mendoza A, Penilla RP, Rodríguez DA (2009) Insecticide resistance and glutathione S-transferases in mosquitoes: a review. Afr J Biotechnol 8(8): 1386-1397.

24. Kumar S, Thomas A, Pillai M (1991) Involvement of mono-oxygenases as a major mechanism of deltamethrin-resistance in larvae of three species of mosquitoes. Indian J Exp Bio 29(4): 379-384.

25. Kasai S, Weerashinghe IS, Shono T (1998) P450 monooxygenases are an important mechanism of permethrin resistance in Culex quinquefasciatus Say larvae. Arch Insect Biochem Physiol 37(1): 47-56.

26. Brooke B, Kloke G, Hunt RH, Koekemoer LL, Tem EA, et al. (2001) Bioassay and biochemical analyses of insecticide resistance in southern African Anopheles funestus (Diptera: Culicidae). Bull Entomol Res 91(04): 265-272

27. Polson KA, Rawlins SC, BrogdonWG, Chadee DD (2011) Characterisation of DDT and Pyrethroid Resistance in Trinidad and Tobago populations of Aedes aegypti. Bull Entomol Res 101(04): 435-441.

28. Enayati AA, Vatandoost H, Ladonni H, Townson H, Hemingway J (2003) Molecular evidence for a kdr-like pyrethroid resistance mechanism in the malaria vector mosquito Anopheles stephensi. Med Vet Entomol 17(2): 138-144.

29. Martinez Torres D, Chevillon C, Brun Barale A, Berge JB, Pasteur N, et al. (1999) Voltage-dependent $\mathrm{Na}+$ channels in pyrethroid-resistant $C x$. pipiens L mosquitoes. Pestic Sci 55(10): 1012-1020.

30. Kasai S, Komagata O, Itokawa K, Shono T, Ng LC, et al. (2014) Mechanisms of pyrethroid resistance in the dengue mosquito vector, Aedes aegypti: target site insensitivity, penetration, and metabolism. PLoS Negl Trop Dis 8(6): e2948.

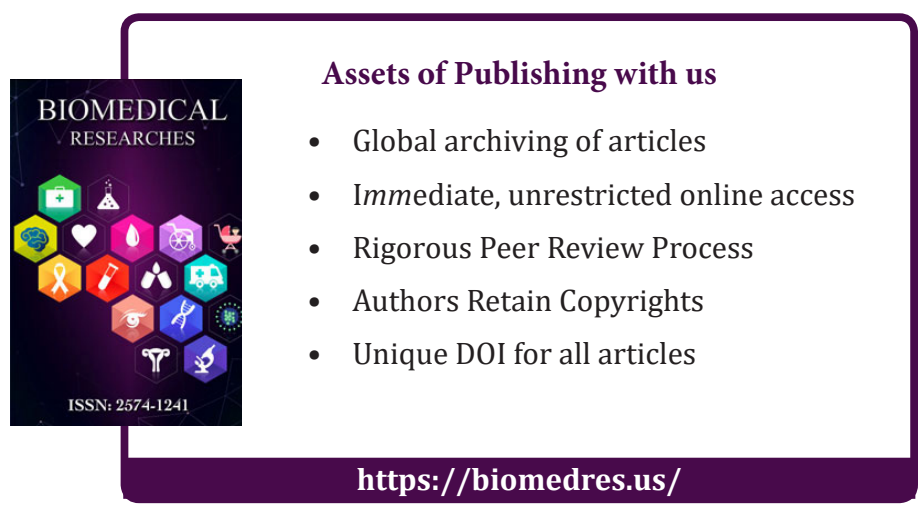

